\title{
Treatment of experimental autoimmune uveoretinitis with different natural compounds
}

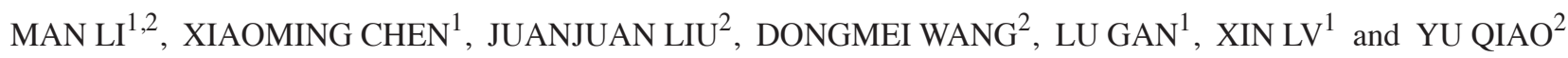 \\ ${ }^{1}$ Department of Ophthalmology, West China Hospital of Sichuan University; \\ ${ }^{2}$ Department of Ophthalmology, AVIC 363 Hospital of Chengdu, Chengdu, Sichuan 610041, P.R. China
}

Received January 11, 2016; Accepted April 8, 2016

DOI: $10.3892 / \mathrm{mmr} .2016 .5096$

\begin{abstract}
Uveitis is an important eye disease that potentially causes loss of sight. Although extensive studies have been conducted on uveitis, the exact pathogenesis remains to be determined. The effects of treatment with natural compounds on an experimental autoimmune uveoretinitis (EAU) rat model were examined in the present study. A total of 25 rats were divided into 5 groups: Alkaloids $(n=5)$, saponins $(n=5)$, flavonoids $(n=5)$, phenols $(n=5)$, and the normal saline group $(n=5)$. The rats in each group were treated with an intraperitoneal injection of proper alkaloids (berberine hydrochloride), saponins (steroidal saponins), flavonoids (baicalein), or phenols (chlorogenic acid) or physiological saline, respectively. The rats' aqueous humour and crystalline lens was then observed under the slit lamp periodically, looking for signs of inflammation. After 2 weeks, the rats were sacrificed and the degree of pathological changes on their eyeballs under different treatment methods were determined using an optical microscope. The expression of the interleukin (IL)-17 gene in the ocular tissues of the rats was assessed via RT-PCR and western blot analysis. Apoptosis on the rats' retinal tissues was detected using flow cytometry. The results showed that rats injected with phenols (chlorogenic acid) had serious ocular vascular dilatation, iris hemorrhage and purulent exudation; those injected with alkaloids (berberine hydrochloride) and flavonoids (baicalein) had a more mild form of inflammation; and those administered saponins (steroidal saponins) had only mild inflammation signs. Following detection of IL-17 mRNA and protein expression levels in the ocular tissues of rats of the five groups, it was found that their expression was lowest in the saponin-treated group and the other differences in expression were all statistically significant $(\mathrm{P}<0.05)$. A comparison with
\end{abstract}

Correspondence to: Dr Xiaoming Chen, Department of Ophthalmology, West China Hospital of Sichuan University, 37 Guoxue lane, Chengdu, Sichuan 610041, P.R. China

E-mail: xiaoming_chen1@163.com

Key words: natural compounds, autoimmune, experimental autoimmune uveoretinitis, fluorescent quantitation other groups revealed that cell apoptosis in the eyes of rats in the saponin group was the most prominent, reflecting a beneficial decrease in the amount of inflammatory cells on the lesion. Based on these findings, natural compounds such as saponins (steroidal saponins), alkaloids (berberine hydrochloride), and flavonoids (baicalein), but not phenols (chlorogenic acid), can inhibit the clinical symptoms of EAU in rats to a certain extent and reduce cell apoptosis. The most promising results in the present study were obtained using steroidal saponins.

\section{Introduction}

Uveitis is a leading cause of visual morbidity and causes approximately 30,000 new cases of legal blindness annually in the United States alone (1). Although uveitis has been previously investigated, the exact pathogenesis remains to be determined (2). It has been proposed that uveitis results mainly from autoimmune reaction defects (1). However, since the exact pathogenesis of uveitis remains to be identified, there are no specific and few effective drugs for treating uveitis. Experimental autoimmune uveoretinitis (EAU) is an attractive animal model for the study of autoimmune uveitis and even other relevant autoimmune diseases that affect humans (3) and it has been recognized as an ideal animal model for drug treatment and assessment. This model has many advantages, including the fact that it is highly reproducible, the clinical manifestations in the rats are easy to detect, and many test results are amenable to quantitative processing.

Certain immunodeficiency syndromes and allergic reactions are caused by excessive immune responses stimulated by endogenous or exogenous antigens that mimic self-antigens (4). The majority of currently available anti-allergy drugs are chemically synthetic drugs characterized by their strong efficacy but also serious side effects (5). Compared with these chemically synthetic drugs, natural drugs are advantageous as they produce fewer side effects (6). Results of previous studies suggested that the mutual effects of quercetin and isoquercetin can reduce the incidence of asthma to some extent; kaempferol, a flavonoid, can inhibit the degranulation of mast cells; and triterpenes can effectively treat pleural hypersensitivity (6).

In the present study, the curative effects of natural compounds on EAU were investigated in a relevant rat model. The findings provide a theoretical and experimental basis for the treatment of EAU. 

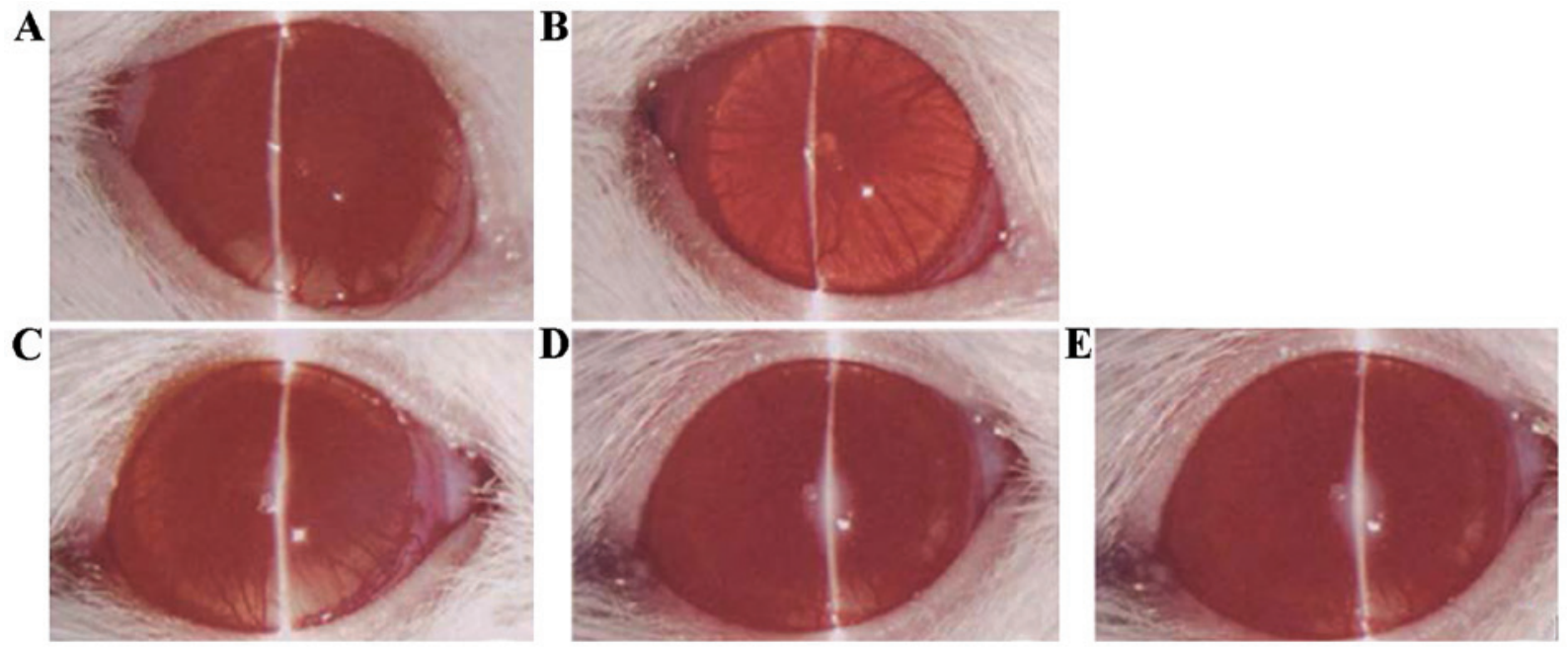

Figure 1. Inflammation in anterior segment observed under slit-lamp microscope (magnification, x100). (A) Saponins, (B) flavonoids, (C) alkaloids, and (D) phenols groups (similar to the normal saline group), and (E) saline group.

\section{Materials and methods}

Animals. A total of 25 EAU rats that were housed and fed in animal cages in West China Hospital were enrolled in our study. The rats were male, ranging from 6 to 8 weeks, with an average weight of $160 \pm 32.5 \mathrm{~g}$. The rats were randomly divided as follows ( $\mathrm{n}=5$ per group): Alkaloids (berberine hydrochloride), saponins (steroidal saponins), flavonoids (baicalein), phenols (chlorogenic acid) and physiological saline groups. The present study was approved by the ethics commitee of the Animal Centre of West China Hospital.

Main reagents. The main reagents used were: Goat anti-rabbit IL-17 primary antibody, and biotinylated donkey anti-sheep ABC immunohistochemistry kit (Roche Diagnostics, Indianapolis, IN, USA); RT-polymerase chain reaction (PCR) reagent (Takara Bio, Inc., Otsu, Japan); and cell apoptosis detection kit (Roche Diagnostics).

Animal grouping and EAU model establishment. EAU models were established in accordance with the methods employed by Oh-i et al (7). The 25 rats were randomly divided into groups as follows ( $\mathrm{n}=5$ per group): alkaloids group $(2 \mathrm{mg} / \mathrm{kg}$ berberine hydrochloride), saponins group $(2.5 \mathrm{mg} / \mathrm{kg}$ steroidal saponins), flavonoids group ( $2.5 \mathrm{mg} / \mathrm{kg}$ baicalein), phenols group ( $2 \mathrm{mg} / \mathrm{kg}$ chlorogenic acid) and physiological saline group ( $2 \mathrm{mg} / \mathrm{kg}$ normal saline). The amounts of the natural compounds injected intraperitoneally were confirmed to be appropriate by preliminary experiments.

Slit lamp microscope observation. The preocular reactions of the rats treated by one of the different natural compounds were observed once a day under a slit-lamp microscope (Hangzhou Medical Science and Technology Company, Hangzhou, China) and their inflammatory reactions were recorded and scored according to the criteria of Kohno et al (8). The criteria were as follows: No inflammation present (0 points), iris congestion and mild retinal vasculitis (1 point), mild fibrous tissue exudation in anterior chamber ( 2 points), moderate exudation and mild empyema in anterior chamber ( 3 points), severe bleeding and empyema in anterior chamber (4 points).

Pathological observation of retina. Following treatment the rats in each group were kept for 2 weeks and were fed as normal during that period of time, prior to injection with $10 \%$ chloral hydrate. The rats were subsequently sacrificed using cervical dislocation. The eye balls were extracted and fixed in a $4 \%$ formaldehyde solution, sliced by routine paraffin sectioning, stained with hematoxylin and eosin, and observed under a microscope (Olympus CX23, Tokyo, Japan). The severity of pathology was evaluated according to the Koho $\mathrm{H}$ evaluation system (8): Normal (0 points), retinal receptor lesion (1-2 points), external granular layer lesions of the retina (3-4 points), retinal internal granular layer lesions (5-6 points), and retinal cellular layer lesions (7 points).

\section{RT-PCR detection and ELISA detection}

Tissue sample RNA extraction. Frozen tissue samples (0.1 g) were taken from liquid nitrogen and allowed to melt on the ice. RNA Plus (0.45 ml) (Takara, Shanghai, China) was added to each sample, then a pre-cooled mortar was used to pulverize the tissues. After transferring to a $15 \mathrm{ml}$ Eppendorf (EP) tube (Axygen, New York, NY, USA), $0.45 \mathrm{ml}$ RNA Plus was used to wash the mortar, which was added to the pulverized tissue sample. Chloroform $(200 \mu \mathrm{l})$ was added into a centrifuge tube and the mix was agitated for $15 \mathrm{sec}$, after which the phases were allowed to separate on ice for $15 \mathrm{~min}$. The tubes were centrifuged at $8,000 \mathrm{xg}$ for $15 \mathrm{~min}$ at $4^{\circ} \mathrm{C}$. The resultant supernatants were then transferred into de-RNase EP tubes (Axygen), the same amount of isopropanol was added to each tube, the tubes were inverted to mix the solutions, and then set aside on ice for $10 \mathrm{~min}$. The tubes were centrifuged at $8,000 \mathrm{xg}$ for $10 \mathrm{~min}$ at $4^{\circ} \mathrm{C}$ and the supernatants were discarded. Then, $750 \mu 175 \%$ ethanol was added to each tube, the tubes were gently mixed by inversion, and then centrifuged at $8,000 \mathrm{x} \mathrm{g}$ for $10 \mathrm{~min}$ at $4^{\circ} \mathrm{C}$. The supernatants were discarded, and any residual ethanol was removed by air drying. Subsequently, de-RNase $\mathrm{H}_{2} \mathrm{O}$ was added, and after measuring the quality 
of the extracted RNA, the samples were preserved for reverse transcription.

$R T-P C R$. PCR was used to detect the expression of $I L-17$ gene, the EAU marker gene. The Oligon 7.0 software (Molecular Biology Insights, Inc., Cascade, CO, USA) was used to design the primers. The primer sequences used were: Upstream primer 5-'CCCATCATTGCAATAGCAGG-3', and downstream primer 5'-GCTCAAACTYCTGCTCCTGA-3'. The length of the expected amplified fragment was $170 \mathrm{bp}$. The parameters used for the fluorescent quantitative PCR reaction system were: SYBR-Green reagent $(5 \mu 1)$, forward primer $(0.5 \mu \mathrm{l})$, reverse primer $(0.5 \mu \mathrm{l})$, and reverse transcriptase product $\left(1 \mu \mathrm{l}, \mathrm{ddH}_{2} \mathrm{O}: 3 \mu \mathrm{l}\right)$. The PCR conditions were $95^{\circ} \mathrm{C}$ for $5 \mathrm{~min}$ and then 40 cycles of $95^{\circ} \mathrm{C}$ for $10 \mathrm{sec}$ denaturation, followed by $60^{\circ} \mathrm{C}$ for $30 \mathrm{sec}$ annealing/extension.

ELISA. ELISA (Roche Diagnostics) was applied to detect the amount of IL-17 protein expression. Surgery was performed strictly in accordance with the protocol of the Roche kit. The absorbance value was measured at $450 \mathrm{~nm}$ after reaction completion and the amount of protein expression was calculated according to a standard protein curve (9).

Apoptosis detection using flow cytometry. In the present study, apoptosis of the cells of rat's retinas treated with different natural compounds were observed using a flow cytometer and operations were performed strictly in accordance with the protocol of the Roche kit.

Western blotting detection. A Roche animal cell protein extraction kit was used to extract the samples of total protein according to the manufacturer's instructions. The primary antibody was mouse anti-human IL-17 monoclonal antibody (Santa Cruz Biotechnology, New York, NY, USA, cat. no.: sc-374218. The secondary antibody was HRP-goat anti-mouse antibody (Santa Cruz Biotechnology, cat. no.: sc-395758). Western blotting was conducted as previously described (7).

Statistical analysis. SPSS 20.0 software (IBM SPSS, Armonk, NY, USA) was used for statistical analyses. Measurement data were shown as mean \pm standard deviation and count data were analyzed by the $\chi^{2}$ test. $\mathrm{P}<0.05$ was considered to indicate statistically significant differences.

\section{Results}

Ocular inflammation. By observing the state of ocular inflammation of rats treated with different natural compounds, it was found that rats in the phenol (chlorogenic acid) and normal saline groups had serious ocular vascular dilatation, iris hemorrhage and purulent exudation; rats in the alkaloid (berberine hydrochloride) and flavonoid (baicalein) groups had slight inflammation; and rats in the saponin (steroidal saponins) group had the slightest inflammation (Fig. 1).

Following a comparison of the clinical scores of the treatment groups, the differences were found to be statistically significant $(\mathrm{F}=6.72 \mathrm{P}=0.003)$. Compared with the scores of the normal saline group, the clinical scores of the saponins (steroidal saponins) group were significantly reduced, and the

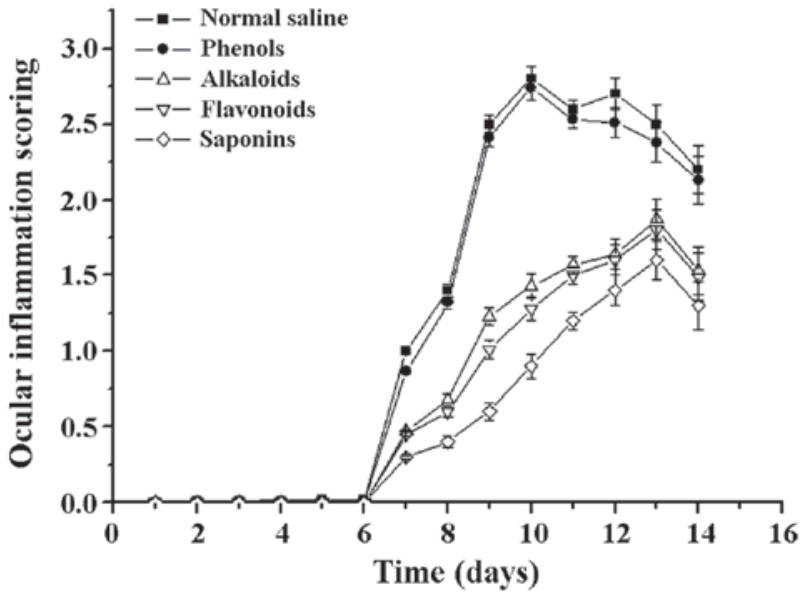

Figure 2. Ocular inflammation scoring under the treatment of different natural compounds.

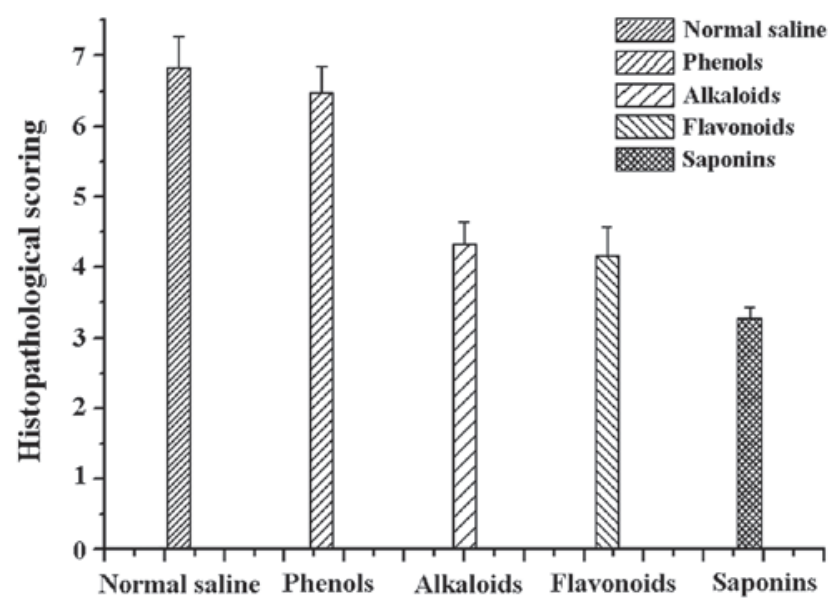

Figure 3. Scoring of retinal pathological examination.

difference was statistically significant $(\mathrm{t}=-0.634 \mathrm{P}=0.002)$. By contrast, the difference between the phenols (chlorogenic acid) and physiological saline groups was not statistically significant $(\mathrm{t}=-0.334 \mathrm{P}=0.538$; Fig. 2).

Histopathological examination. The ocular pathology scoring of rats differed between groups. The rats on saponins had the smallest score (3.28 \pm 0.159$)$, which was in agreement with the above results. Rats administered alkaloids $(4.32 \pm 0.328)$, and flavonoids (4.17 \pm 0.401$)$ yielded similar results. Rats administered phenols $(6.47 \pm 0.369)$ and normal saline $(6.83 \pm 0.437)$ had the most retinal damage. There was no statistical difference between the normal saline and phenols group $(\mathrm{F}=12.76$ $\mathrm{P}=0.000)$. The differences between the normal saline group and the remaining three groups were statistically significant $(\mathrm{t}=-1.48, \mathrm{P}=0.04)$. Specifically, the difference between the normal saline and saponins group was statistically significant ( $\mathrm{t}=-1.27, \mathrm{P}=0.01$; Fig. 3).

RT-PCR, ELISA, and western blot analysis. Previous studies have indicated that IL-17 expression is closely associated with the pathogenesis and deterioration of EAU, and IL-17 can be used as a marker to identify the severity of uveitis, rheumatoid 
Table I. Cell apoptosis of different treatment groups (mean \pm standard deviation, $\mathrm{n}=12$ ).

\begin{tabular}{lc}
\hline Group & Cell apoptotic rate $(\%)$ \\
\hline Normal saline & $2.13 \pm 0.017$ \\
Phenols (chlorogenic acid) & $54.8 \pm 0.042^{\mathrm{a}}$ \\
Alkaloids (berberine hydrochloride) & $65.7 \pm 0.012^{\mathrm{a}}$ \\
Flavonoids (baicalein) & $74.2 \pm 0.068^{\mathrm{a}}$ \\
Saponins (steroidal saponins) & $83.4 \pm 0.037^{\mathrm{b}}$ \\
\hline
\end{tabular}

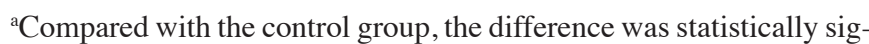
nificant $(\mathrm{P}<0.05)$. ${ }^{\mathrm{b}}$ Compared with the control group, the difference was statistically significant $(\mathrm{P}<0.01)$.

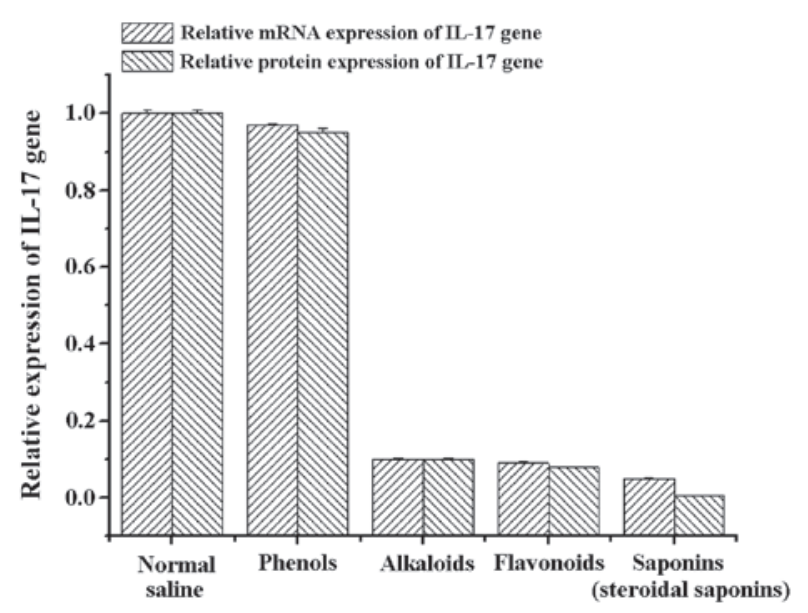

Figure 4. mRNA and protein expression of interleukin (IL)-17 in the lesion tissues under treatment with different natural compounds.

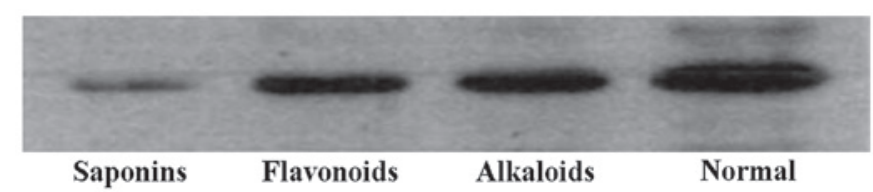

Figure 5. Western blot analysis results of interleukin-17 protein in the lesion tissues of the different treatment groups.

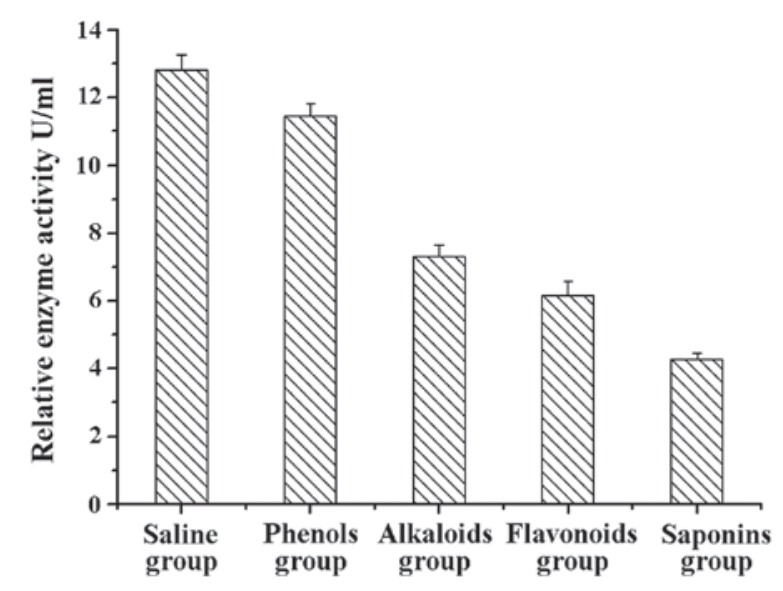

Figure 6. ELISA result of the interleukin-17 protein in the lesion tissues of the different treatment groups.
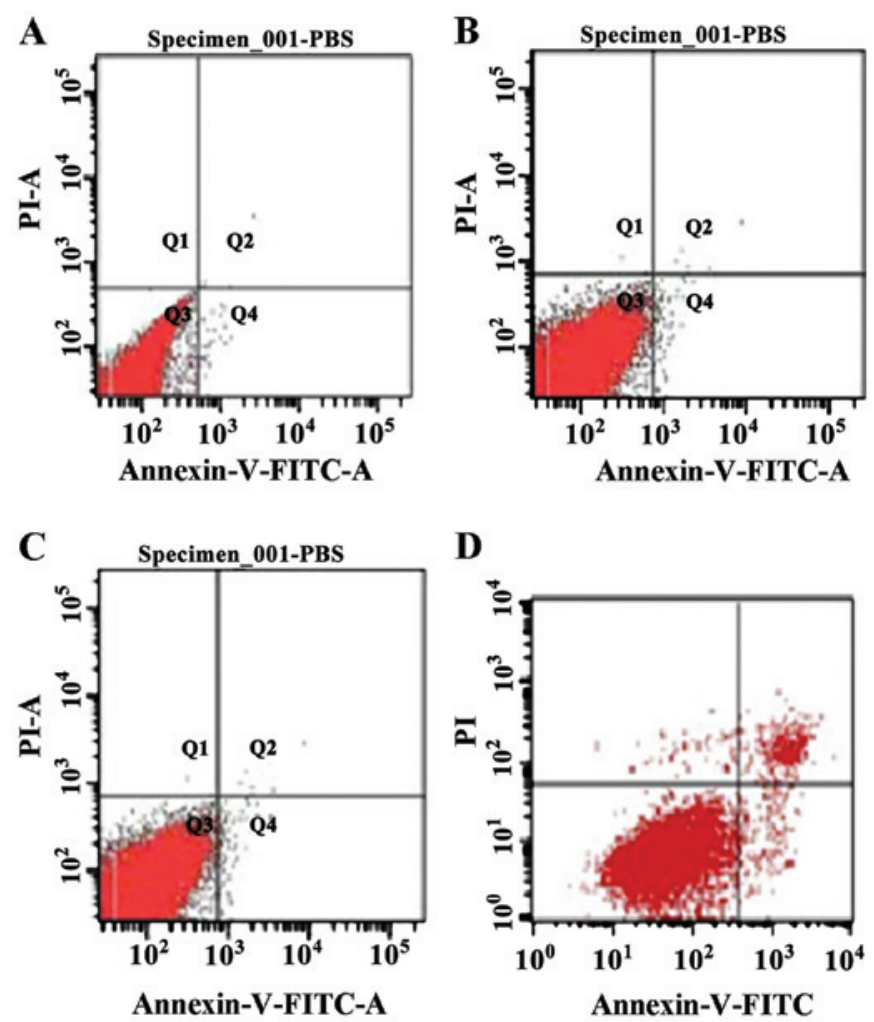

Annexin-V-FITC-A

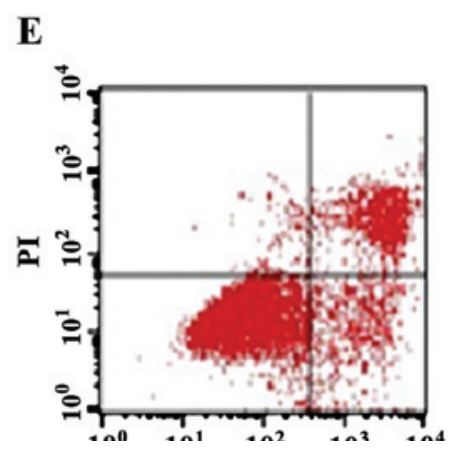

Figure 7. Flow cytometry plots for detecting apoptosis in cells derived from eyeballs in different treatment groups: (A) Saline, (B) phenols, (C) alkaloids, (D) flavonoids, and (E) saponins groups.

arthritis, multiple sclerosis, systemic lupus erythematosus and asthma (10). Therefore, in the present study, we extracted RNA from the retinal tissues of rats in the different treatment groups and detected the mRNA and protein expression of IL-17. Detection results showed that the amount of mRNA and protein expression of $I L-17$ genes in the alkaloids (berberine hydrochloride) group, saponins (steroidal saponins) group and flavonoids (baicalein) group was significantly lower than the normal saline and phenols (chlorogenic acid) groups (Fig. 4). These results were further supported by western blot analysis and ELISA with anti-IL-17 antibodies, indicating that the the three natural compounds may effectively reduce the amount of IL-17 protein expression (Figs. 5 and 6).

Detection of cell apoptosis. A search was conducted for apoptosis markers on the ocular cells of rats in the different treatment groups using flow cytometry. The results showed that cell apoptosis in the alkaloids, saponins, and flavonoids 
groups was significantly higher than that in the control group (normal saline group) (Table I), indicating that these natural compounds can promote apoptosis of relevant inflammatory cells, thereby exerting a beneficial effect for the rehabilitation of inflammation (Fig. 7).

\section{Discussion}

Uveitis is an important eye disease that may cause blindness. It has been extensively investigated, however, its exact pathogenesis remains to be determined (10), resulting in a lack of specifically efficient drugs for its treatment $(11,12)$. Arsenic trioxide has been shown to be effective in the treatment of acute promyelocytic leukemia and other tumors, and more recently investigators tested it against EAU, via intraperitoneal injection, with promising results, albeit a high incidence of adverse effects (13). Other investigators have employed cyclophosphamide because of its inhibitory effects on $\mathrm{CD}$ lymphocytes (supressing humoral immunity and IL-17 expression) (14). However, the results have shown that although cyclophosphamide suppresses humoral immunity and reduces the gene expression of peripheral IL-17, its effects on EAU are only mildy beneficial (15). At least one group of investigators has used traditional drugs that seem to act more directly on the eye, improving the clinical syndromes associated with the ocular inflammation caused by uvetis, albeit those treatments do not provide successful treatment for the disease (16).

Many natural compounds can be effective for the treatment of certain anaphylactic diseases (17). Compared with conventional drugs, the negative effects of natural compounds are less prominent. In a previous study, volatile oil of Angelica, a natural compound, effectively treated passive cutaneous anaphylaxis and parietal periosteum hypertrophy in rats (18). A different study confirmed that the volatile oil of schizonepeta indirectly inhibited inflammation by suppressing the metabolic products of arachidonic acid epoxidase and lipoxidase (19). Based on the abovementioned results, the present study was carried out to examine the curative effects of natural compounds on uveitis $(20,21)$. The results show that natural compounds were more effective for EAU treatment than normal saline. However, the curative effects of the different natural compounds tested varied. Of the four types of natural compounds, the curative effects of saponins were the most promising, with alkaloids and flavonoids providing the second best results, while phenols were basically ineffective. Such differences are probably associated with the different compositions of the tested compounds. Terpenoids are the major component of saponins. Recent findings have shown the effectiveness of certain terpenoids for dealing with inflammation (18). Additionally, the major components of phenols are alkaline substances, which probably cause many toxic effects on tissues. The results of the present study require further investigation with regard to the use of saponins and terpenoids for the treatment of uveitis.

\section{References}

1. Witkowski L, Cywinska A, Paschalis-Trela K, Crisman M and Kita J: Multiple etiologies of equine recurrent uveitis - A natural model for human autoimmune uveitis: A brief review. Comp Immunol Microbiol Infect Dis 44: 14-20, 2016.
2. Wakefield D and Abi-Hanna D: HLA antigens and their significance in the pathogenesis of anterior uveitis: A mini review. Curr Eye Res 5: 465-473, 1986.

3. Maca SM, Amirian A, Prause C, Gruber K, Mejdoubi L and Barisani-Asenbauer T: Understanding the impact of uveitis on health-related quality of life in adolescents. Acta Ophthalmol 91: e219-e224, 2013.

4. Berthet F, Le Deist F, Duliege AM, Griscelli C and Fischer A: Clinical consequences and treatment of primary immunodeficiency syndromes characterized by functional $\mathrm{T}$ and $\mathrm{B}$ lymphocyte anomalies (combined immune deficiency). Pediatrics 93: 265-270, 1994.

5. Zhang Q, Lu J, Liang X and Zhi L: The level and clinical significance of serum IL-17 in patients with systemic lupus erythematosus. Xibao Yu Fenzi Mianyixue Zazhi 28: 301-303, 2012 (In Chinese).

6. Cui Y, Wang G, Li Y, Wang Y, Wang X and Bi H: Optical coherence tomography and histopathology of macular uveitis. Optom Vis Sci 91: 1335-1342, 2014.

7. Oh-i K, Keino H, Goto H, Yamakawa N, Murase K, Usui Y, Kezuka T, Sakai J, Takeuchi M and Usui M: Intravitreal injection of Tacrolimus (FK506) suppresses ongoing experimental autoimmune uveoretinitis in Rats. Br J Ophthalmol 91: 237-242, 2007.

8. Kohno H, Sakai T, Saito S, Okano K and Kitahara K: Treatment of experimental autoimmune uveoretinitis with atorvastatin and lovastatin. Exp Eye Res 84: 569-576, 2007.

9. Kezic JM, Glant TT, Rosenbaum JT and Rosenzweig HL: Neutralization of IL-17 ameliorates uveitis but damages photoreceptors in a murine model of spondyloarthritis. Arthritis Res Ther 14: R18, 2012.

10. Chi W, Zhu X, Yang P, Liu X, Lin X, Zhou H, Huang X and Kijlstra A: Upregulated IL-23 and IL-17 in Behçet patients with active uveitis. Invest Ophthalmol Vis Sci 49: 3058-3064, 2008.

11. Sng CC, Ang M and Barton K: Uveitis and glaucoma: New insights in the pathogenesis and treatment. Prog Brain Res 221: 243-269, 2015

12. Wells JM and Smith JR: Uveitis in juvenile idiopathic arthritis: Recent therapeutic advances. Ophthalmic Res 54: 124-127, 2015.

13. Zou ZP, Qiang FY, Zhang T, Sun L, Jia T, Zhu XC and Xu H: Treatment of experimental autoimmune uveitis in rats with arsenic trioxide. Chin Ophthalmic Res 28: 306-310, 2010.

14. Klímová A, Seidler Štanogová P, Heissigerová J, Svozílková $\mathrm{P}$ and Kučera T: Mycophenolate mofetil and cyclophosphamide treatments suppress inflammation intensity in an experimental model of autoimmune uveitis. Folia Biol (Praha) 60: 228-234, 2014.

15. Tang Y and Cai L: Research progress of regulatory $\mathrm{T}$ cells and uveitis. Int J Ophthalmol 11: 450-452, 2011.

16. Li J, Chen X, Liu Z, Yue Q and Liu H: Expression of Th17 cytokines in skin lesions of patients with psoriasis. J Huazhong Univ Sci Technolog Med Sci 27: 330-332, 2007.

17. de Smet MD, Taylor SRJ, Bodaghi B, Miserocchi E, Murray PI, Pleyer U, Zierhut M, Barisani-Asenbauer T, LeHoang P and Lightman S: Understanding uveitis: The impact of research on visual outcomes. Prog Retin Eye Res 30: 452-470, 2011.

18. Mira A, Tanaka A, Tateyama Y, Kondo R and Shimizu K: Comparative biological study of roots, stems, leaves, and seeds of Angelica shikokiana Makino. J Ethnopharmacol 148: 980-987, 2013.

19. Byun MW: Schizonepeta tenuifolia ethanol extract exerts anti-inflammatory activity through the inhibition of TLR4 signaling in lipopolysaccharide-stimulated macrophage cells. J Med Food 17: 350-356, 2014

20. Jäger A and Kuchroo VK: Effector and regulatory T-cell subsets in autoimmunity and tissue inflammation. Scand J Immunol 72: 173-184, 2010.

21. Tang K, Si J-K, Guo D-D, Cui Y, Du YX, Pan XM and Bi HS: Ranibizumab alone or in combination with photodynamic therapy vs photodynamic therapy for polypoidal choroidal vasculopathy: A systematic review and Meta-analysis. Int J Ophthalmol 8: 1056-1066, 2015. 